\title{
Postoperative Analgesic Efficiency of Ultrasound Guided Transversus Abdominus Plane Block Using Different Concentrations of Bupivacaine in Inguinal Hernia Repair
}

Sinem Keskin Kayalar, Nurten Bakan, Mehtap Ozdemir, Mehmet Salim Akdemir

University of Health Science FSM-Sancaktepe Training and Research Hospital, University of Health Science Umraniye Training and

Research Hospital, Istanbul, Turkey

\section{Backgrounds and goal of study:}

Effective postoperative analgesia is very important in reducing postoperative morbidity, accelarating recovery and avoding chronic postoperative pain.

Ultrasound guided transversus abdominis plane (TAP) block is done as a part of multimodal analgesia for pain relief after inguinal hernia surgeries.

This study was conducted to evaluate postoperative analgesic efficacy of unilateral TAP Block using different concentration of bupivacaine in patients undergoing inguinal hernia surgeries.

\section{Material and Methods:}

After ethical approval and patient informed consent, seventy-five patients scheduled for open inguinal hernia repair surgery were divided into three equal groups.

Group-I (Gl) unilateral TAP block with bupivacaine 0.25\% $20 \mathrm{ml}$,

Group-II (GII) unilateral TAP block with bupivacaine $0.125 \%$ $20 \mathrm{ml}$,

Group-III (GIII) no TAP block.

The patient's haemorespiratuar parameters were normal. After induction, same general anesthetic technique was chosen for all groups.

TAP Blocks were performed with ultrasound guidance, in the midaxillary line.

Plain bupivacaine $0.25 \%$ or $0.125 \%, 20 \mathrm{ml}$ were applied via 22 gauge neddle between internal oblique and transversus abdominis muscles .

Postoperative pain scores were evaluated with visual analog scale (VAS) at 30th and 60th min on recovery room, at 2., 4., 8., 12. and 24th hour in the ward.

An additional and a rescue analgesics were applied when VAS scores $\geq 4$.
First analgesic application time, the number of additional and rescue analgesics, postoperative nausea-vomiting and patient satisfaction were recorded.

\section{Results and Discussions:}

MAP, HR, SpO2, EtCO2 values were similar intraoperatively.

The postoperative pain scores were statistically lower in Gl and GII than GIII in recovery room and in the ward $(p<0.05)$ (Figure 1).

The patient number of additional analgesic applied was significantly higher in GIII than GI and GII ( $p<0.05)$ (Figure 2). The first analgesic application time was longer in $\mathrm{Gl}$ and GII compared with GIII $(p<0.05)$. The number of rescue analgesic was significantly higher in GIII compared to GI and GII $(p<0.05)$ (Table 1).

The patients in the GIII were less satisfacted than the GI and GII $(p<0.05)$ (Figure 3).

All parameters were similar in $\mathrm{Gl}$ and GII ( $p>0.05)$.

There was no difference in the incidence of nauseavomiting.

Table1: First analgesic time, additional number of analgesic and total rescue analgesic according to groups

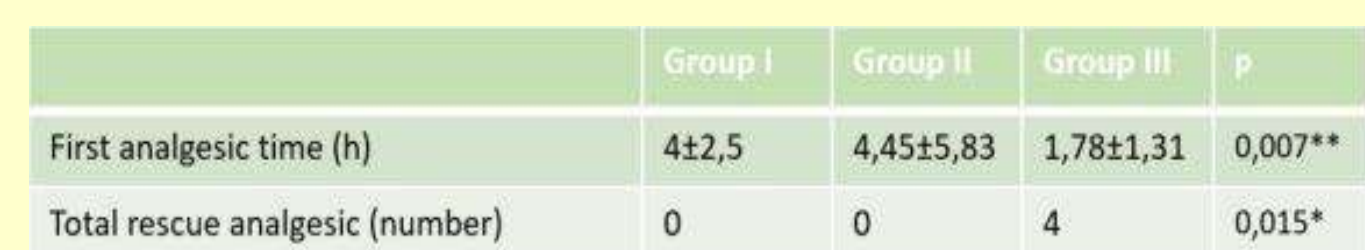

\section{Conclusions:}

TAP Block performed under ultrasound guidance before the surgical incision provided effective and prolonged postoperative analgesia.

Using different concentrations of bupivacaine $(0.125 \%$ and $0.25 \%$ ) produced similar postoperative pain scores, incidence of nausea-vomiting, higher patient satisfaction and provided considerable reduction of postoperative analgesic requirements.
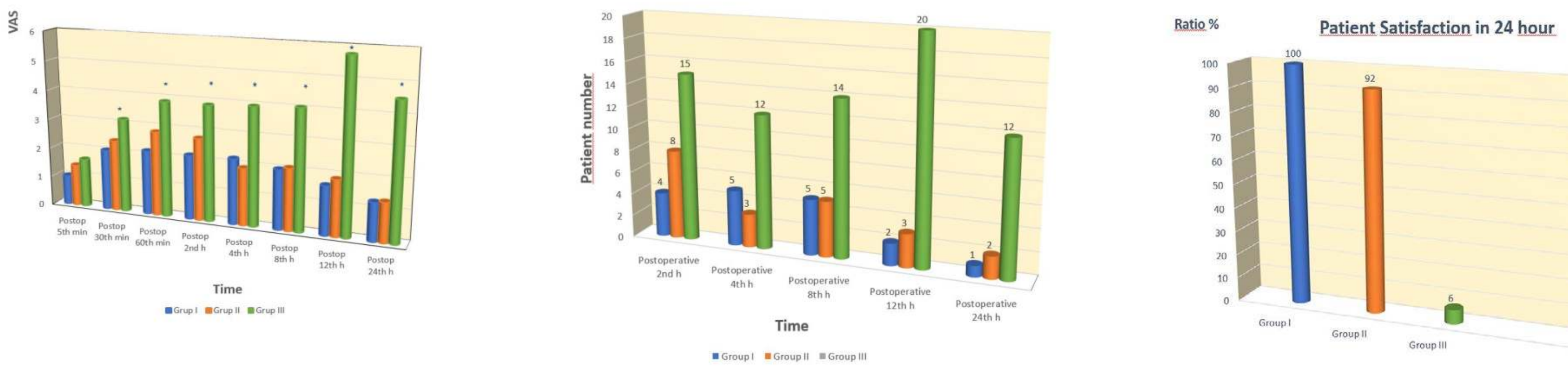